\title{
Distortion Corrections for the Many Beam Fabry Perot Velocimeter
}

\author{
G. Avara, L. Collins, A. Rivera
}

\section{March 21, 2001}

U.S. Department of Energy

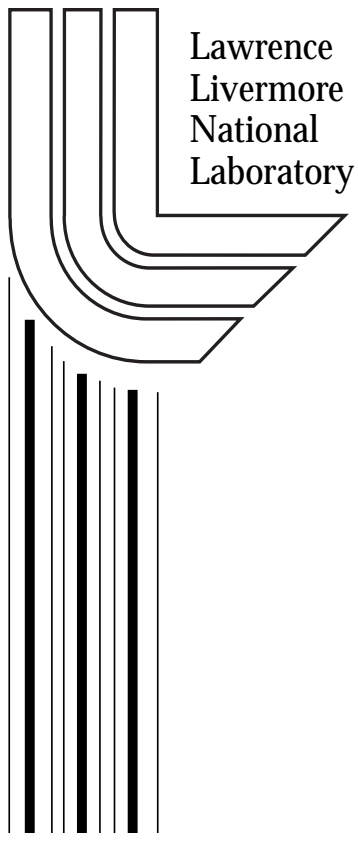




\section{DISCLAIMER}

This document was prepared as an account of work sponsored by an agency of the United States Government. Neither the United States Government nor the University of California nor any of their employees, makes any warranty, express or implied, or assumes any legal liability or responsibility for the accuracy, completeness, or usefulness of any information, apparatus, product, or process disclosed, or represents that its use would not infringe privately owned rights. Reference herein to any specific commercial product, process, or service by trade name, trademark, manufacturer, or otherwise, does not necessarily constitute or imply its endorsement, recommendation, or favoring by the United States Government or the University of California. The views and opinions of authors expressed herein do not necessarily state or reflect those of the United States Government or the University of California, and shall not be used for advertising or product endorsement purposes.

This work was performed under the auspices of the U. S. Department of Energy by the University of California, Lawrence Livermore National Laboratory under Contract No. W-7405-Eng-48.

This report has been reproduced directly from the best available copy.

Available electronically at http://www.doc.gov/bridge

Available for a processing fee to U.S. Department of Energy

And its contractors in paper from

U.S. Department of Energy

Office of Scientific and Technical Information

P.O. Box 62

Oak Ridge, TN 37831-0062

Telephone: (865) 576-8401

Facsimile: (865) 576-5728

E-mail: reports@adonis.osti.gov

Available for the sale to the public from

U.S. Department of Commerce

National Technical Information Service

5285 Port Royal Road

Springfield, VA 22161

Telephone: (800) 553-6847

Facsimile: (703) 605-6900

E-mail: orders@ntis.fedworld.gov

Online ordering: http://www.ntis.gov/ordering.htm

OR

Lawrence Livermore National Laboratory

Technical Information Department's Digital Library

http://www.llnl.gov/tid/Library.html 
UCRL-ID-143225

Distribution Category UC-405

\title{
Distortion Corrections for the Many Beam Fabry Perot Velocimeter
}

\author{
George Avara \\ Leland Collins \\ Anthony Rivera
}

Manuscript date: March 21, 2001 


\section{DISCLAIMER}

This document was prepared as an account of work sponsored by an agency of the United States Government. Neither the United States Government nor the University of California nor any of their employees, makes any warranty, express or implied, or assumes any legal liability or responsibility for the accuracy, completeness, or usefulness of any information, apparatus, product, or process disclosed, or represents that its use would not infringe privately owned rights. Reference herein to any specific commercial product, process, or service by trade name, trademark, manufacturer, or otherwise, does not necessarily constitute or imply its endorsement, recommendation, or favoring by the United States Government or the University of California. The views and opinions of authors expressed herein do not necessarily state or reflect those of the United States Government or the University of California, and shall not be used for advertising or product endorsement purposes.

This work was performed under the auspices of the U. S. Department of Energy by the University of California, Lawrence Livermore National Laboratory under Contract No. W-7405-Eng-48.

This report has been reproduced directly from the best available copy.

Available electronically at http://www.doc.gov/bridge

Available for a processing fee to U.S. Department of Energy

And its contractors in paper from

U.S. Department of Energy

Office of Scientific and Technical Information

P.O. Box 62

Oak Ridge, TN 37831-0062

Telephone: (865) 576-8401

Facsimile: (865) 576-5728

E-mail: reports@adonis.osti.gov

Available for the sale to the public from

U.S. Department of Commerce

National Technical Information Service

5285 Port Royal Road

Springfield, VA 22161

Telephone: (800) 553-6847

Facsimile: (703) 605-6900

E-mail: orders@ntis.fedworld.gov

Online ordering: http://www.ntis.gov/ordering.htm

OR

Lawrence Livermore National Laboratory

Technical Information Department's Digital Library 
http://www.llnl.gov/tid/Library.html 


\title{
Distortion Corrections for the Many Beam Fabry Perot Velocimeter
}

\begin{abstract}
Graphical curves and text tables are presented that map out time and space distortions for data obtained from film records of the Many Beam Fabry Perot Velocimeter. Effective distortion corrections extracted from these mappings can be applied to upcoming velocimetry experiments, but only with limited success over periods of a year or more into the future. A method of using three fiducials to provide fresh time and space distortion data on each film record is presented as a more reliable procedure to correct distortions to an acceptable level of accuracy.
\end{abstract}

\section{Introduction}

To determine velocities of fast moving objects, film records of Doppler shifted interference fringes are obtained from the surface reflected laser light. ${ }^{1}$ Experiments performed at the Lawrence Livermore National Laboratory (LLNL) utilize a Many Beam Fabry Perot Velocimeter to acquire velocities from various positions on a given surface. ${ }^{2}$ Several of these five beam velocimeters are used at LLNL diagnostic facilities at Livermore and Nevada. Laser beams that reflect from five separate surface positions are multiplexed through a single interferometer, and the associated optics (see Fig. 1), to form five interference fringes which are recorded on separate electronic streak cameras. The recorded images are distorted along the sweep direction of the streak (time) and vertical to the sweep (space). If distortions are not corrected out of the data, errors in timing and velocity measurements will result. Most of the distortion is caused by an electronic streak camera and is dynamic (varies with the camera sweep time).

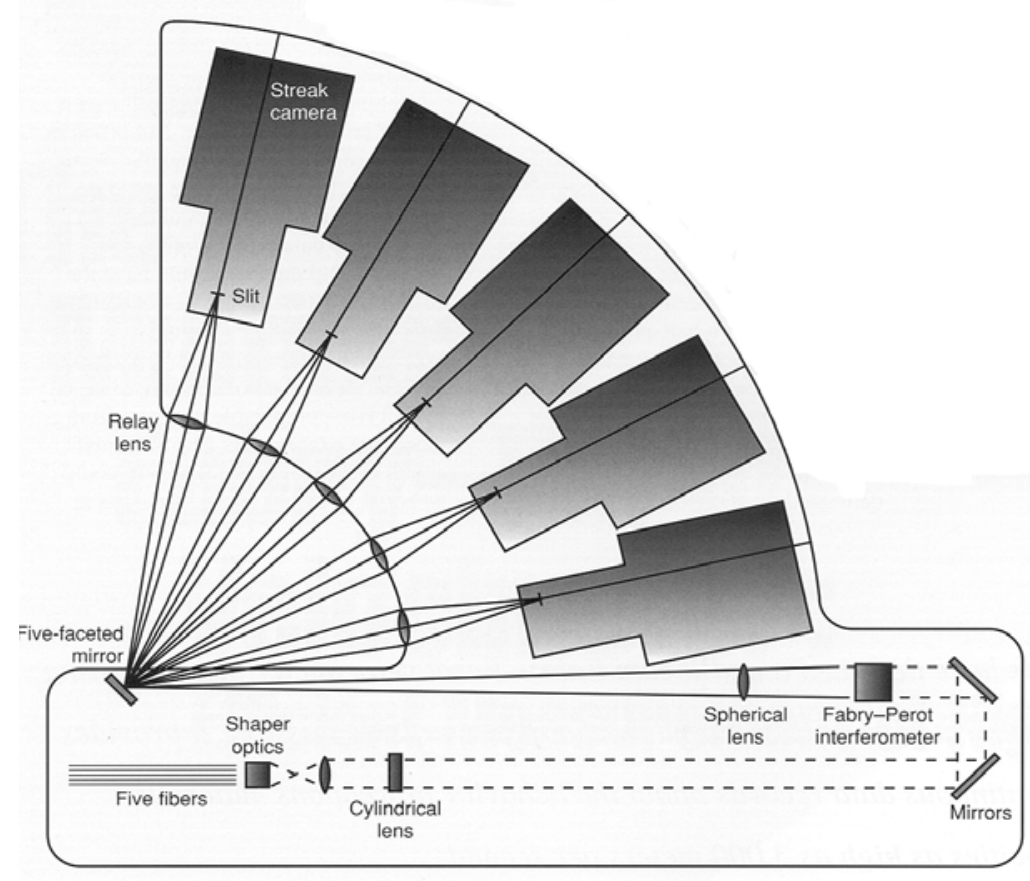

Figure 1. Many Beam Velocimeter 


\section{Distortion Measurements}

When a precision equally spaced ruling is placed at the output focal plane of the shaper optics (Fig. 1), equally spaced bars are imaged through the optics and onto the streak camera slit. Both the cylinder lens and the interferometer are moved out of the optical path for this measurement. The ruling is illuminated with laser light that is injected into the input end of the shaper optics. The top and bottom time fiducials are left intact so that only the inner region of the slit transmits light from the ruling. Light transit times are equal for the fiducials and ruling. Contributions from the glass optical components and the electronic streak camera combine to distort the image at the film plane. If source light from the laser is acoustically modulated then a film record is produced as shown in Fig. 2. These records can be analyzed for time distortions.

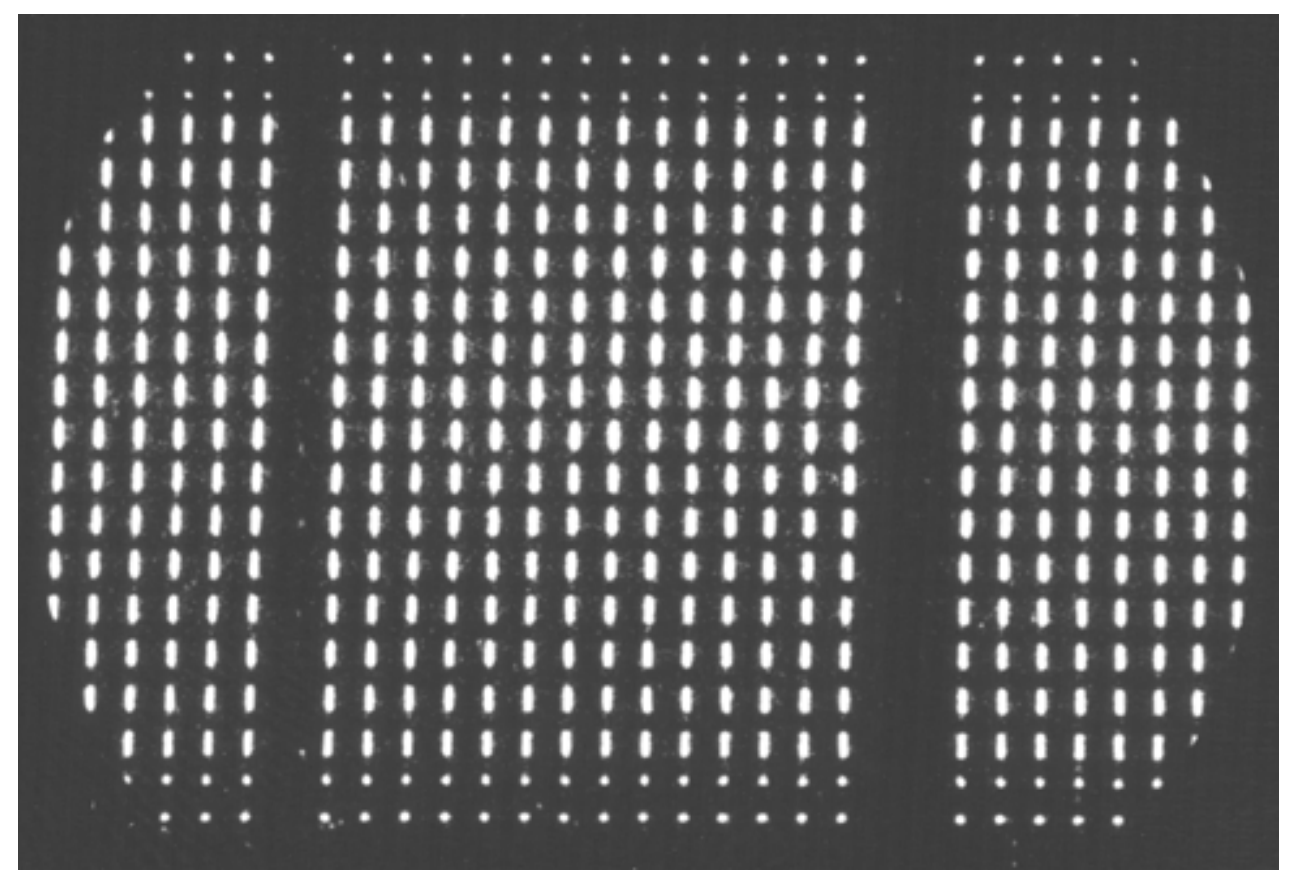

Figure 2. Time Distortion Measurement Record

A large part of time distortions resides in the skew between the top and bottom of a record. Once that skew is removed, an average of quadratic (or cubic) curve fits to the top and bottom time fiducials are currently used for correcting the remaining middle data. However, we can make corrections that are more precise by measuring all of the distortions between the top and bottom fiducials. A problem with this full map approach is that the map corrections become less and less precise over a single year and are ineffective after several years. It is also time consuming and expensive to map out these distortions for each experiment. We have tested out the usefulness of adding a third fiducial (fid) half way between the top and bottom fids. This method works well provided that we use the middle fid to correct from the middle region to the top (or bottom) fid using a gradient formula. This formula uses interpolation so that the middle fid correction curve weakens linearly as the correction zones move from the middle to the top (or to the bottom) time fiducial. Remember that the top and bottom fiducials have already been corrected by the first curve fit routine. Table-1 and table- 2 list the results and compare the different correction methods discussed. 


\begin{tabular}{|c|c|c|c|c|c|c|c|c|c|}
\hline \multirow[t]{2}{*}{$\begin{array}{l}\text { CAMERA } \\
\text { SER. NO. }\end{array}$} & $\begin{array}{c}\text { CAMERA } \\
\text { SWEEP }\end{array}$ & \multicolumn{2}{|c|}{$\begin{array}{c}\text { NO } \\
\text { CORR }\end{array}$} & \multicolumn{2}{|c|}{ TOP /BOT } & \multicolumn{2}{|c|}{$\begin{array}{l}\text { THREE } \\
\text { FIDS }\end{array}$} & \multicolumn{2}{|l|}{ FULL } \\
\hline & Microsec. & \multicolumn{2}{|c|}{ Microsec. } & \multicolumn{2}{|c|}{ Microsec. } & \multicolumn{2}{|c|}{ Microsec. } & \multicolumn{2}{|c|}{ Microsec. } \\
\hline DS1-2 & 8 & 0.041 & $0.6 \%$ & 0.020 & $0.3 \%$ & 0.015 & $0.2 \%$ & 0.009 & $0.1 \%$ \\
\hline DS1-4 & 8 & 0.029 & $0.5 \%$ & 0.023 & $0.4 \%$ & 0.016 & $0.3 \%$ & 0.011 & $0.2 \%$ \\
\hline DS1-5 & 8 & 0.040 & $0.6 \%$ & 0.015 & $0.2 \%$ & 0.012 & $0.2 \%$ & 0.009 & $0.1 \%$ \\
\hline DS2-1 & 8 & 0.052 & $0.8 \%$ & 0.031 & $0.5 \%$ & 0.017 & $0.3 \%$ & 0.009 & $0.1 \%$ \\
\hline DS2-3 & 8 & 0.032 & $0.5 \%$ & 0.020 & $0.3 \%$ & 0.016 & $0.3 \%$ & 0.010 & $0.2 \%$ \\
\hline DS2-5 & 8 & 0.069 & $1.1 \%$ & 0.020 & $0.3 \%$ & 0.018 & $0.3 \%$ & 0.011 & $0.2 \%$ \\
\hline DS2-6 & 8 & 0.032 & $0.5 \%$ & 0.015 & $0.2 \%$ & 0.012 & $0.2 \%$ & 0.008 & $0.1 \%$ \\
\hline DS2-7 & 8 & 0.021 & $0.3 \%$ & 0.013 & $0.2 \%$ & 0.013 & $0.2 \%$ & 0.009 & $0.1 \%$ \\
\hline DS2-8 & 8 & 0.035 & $0.5 \%$ & 0.039 & $0.6 \%$ & 0.022 & $0.3 \%$ & 0.012 & $0.2 \%$ \\
\hline DS2-10 & 8 & 0.026 & $0.4 \%$ & 0.010 & $0.2 \%$ & 0.011 & $0.2 \%$ & 0.007 & $0.1 \%$ \\
\hline Average & & 0.038 & $0.59 \%$ & 0.021 & $0.32 \%$ & 0.015 & $0.24 \%$ & 0.010 & $0.15 \%$ \\
\hline Std Dev & & 0.014 & $0.22 \%$ & 0.009 & $0.14 \%$ & 0.003 & $0.05 \%$ & 0.002 & $0.02 \%$ \\
\hline 95\% Error & & 0.066 & $1.03 \%$ & 0.038 & $0.59 \%$ & 0.022 & $0.34 \%$ & 0.013 & $0.20 \%$ \\
\hline DS1-2 & 40 & 0.245 & $0.8 \%$ & 0.128 & $0.4 \%$ & 0.085 & $0.3 \%$ & 0.040 & $0.1 \%$ \\
\hline DS1-4 & 40 & 0.133 & $0.4 \%$ & 0.118 & $0.4 \%$ & 0.091 & $0.3 \%$ & 0.041 & $0.1 \%$ \\
\hline DS1-5 & 40 & 0.268 & $0.8 \%$ & 0.126 & $0.4 \%$ & 0.060 & $0.2 \%$ & 0.055 & $0.2 \%$ \\
\hline DS2-1 & 40 & 0.269 & $0.8 \%$ & 0.153 & $0.5 \%$ & 0.098 & $0.3 \%$ & 0.049 & $0.2 \%$ \\
\hline DS2-3 & 40 & 0.185 & $0.6 \%$ & 0.102 & $0.3 \%$ & 0.065 & $0.2 \%$ & 0.043 & $0.1 \%$ \\
\hline DS2-5 & 40 & 0.300 & $0.9 \%$ & 0.115 & $0.4 \%$ & 0.088 & $0.3 \%$ & 0.070 & $0.2 \%$ \\
\hline DS2-6 & 40 & 0.212 & $0.7 \%$ & 0.100 & $0.3 \%$ & 0.071 & $0.2 \%$ & 0.037 & $0.1 \%$ \\
\hline DS2-7 & 40 & 0.275 & $0.9 \%$ & 0.075 & $0.2 \%$ & 0.065 & $0.2 \%$ & 0.042 & $0.1 \%$ \\
\hline DS2-8 & 40 & 0.173 & $0.5 \%$ & 0.174 & $0.5 \%$ & 0.112 & $0.4 \%$ & 0.044 & $0.1 \%$ \\
\hline DS2-10 & 40 & 0.258 & $0.8 \%$ & 0.061 & $0.2 \%$ & 0.056 & $0.2 \%$ & 0.041 & $0.1 \%$ \\
\hline Average & & 0.232 & $0.72 \%$ & 0.115 & $0.36 \%$ & 0.079 & $0.25 \%$ & 0.046 & $0.14 \%$ \\
\hline Std Dev & & 0.054 & $0.17 \%$ & 0.034 & $0.10 \%$ & 0.018 & $0.06 \%$ & 0.010 & $0.03 \%$ \\
\hline 95\% Error & & 0.339 & $1.06 \%$ & 0.182 & $0.57 \%$ & 0.116 & $0.36 \%$ & 0.066 & $0.21 \%$ \\
\hline
\end{tabular}

Table-1: Time Distortion Errors after Corrections for Site $\mathbf{3 0 0}$ Cameras $\%$ Is percent of actual record length (Actual $=80 \%$ of Sweep Setting).

The $95 \%$ error is average error plus two standard deviations. 


\begin{tabular}{|c|c|c|c|c|c|c|c|c|c|}
\hline \multirow[t]{2}{*}{$\begin{array}{l}\text { CAMERA } \\
\text { SER. NO. }\end{array}$} & $\begin{array}{c}\text { CAMERA } \\
\text { SWEEP }\end{array}$ & \multicolumn{2}{|c|}{$\begin{array}{c}\text { NO } \\
\text { CORR }\end{array}$} & \multicolumn{2}{|c|}{$\begin{array}{l}\text { TOP /BOT } \\
\text { FIDS }\end{array}$} & \multicolumn{2}{|c|}{$\begin{array}{c}\text { THREE } \\
\text { FIDS }\end{array}$} & \multicolumn{2}{|l|}{$\begin{array}{l}\text { FULL } \\
\text { MAP }\end{array}$} \\
\hline & Microsec. & \multicolumn{2}{|c|}{ Microsec. } & \multicolumn{2}{|c|}{ Microsec. } & \multicolumn{2}{|c|}{ Microsec. } & \multicolumn{2}{|c|}{ Microsec. } \\
\hline DS2-11 & 8 & 0.063 & $1.0 \%$ & 0.024 & $0.4 \%$ & 0.012 & $0.2 \%$ & 0.009 & $0.1 \%$ \\
\hline DS2-12 & 8 & 0.072 & $1.1 \%$ & 0.029 & $0.5 \%$ & 0.014 & $0.2 \%$ & 0.009 & $0.1 \%$ \\
\hline DS2-13 & 8 & 0.067 & $1.0 \%$ & 0.036 & $0.6 \%$ & 0.018 & $0.3 \%$ & 0.012 & $0.2 \%$ \\
\hline DS2-14 & 8 & 0.068 & $1.1 \%$ & 0.037 & $0.6 \%$ & 0.017 & $0.3 \%$ & 0.010 & $0.2 \%$ \\
\hline DS2-15 & 8 & 0.075 & $1.2 \%$ & 0.032 & $0.5 \%$ & 0.025 & $0.4 \%$ & 0.013 & $0.2 \%$ \\
\hline Average & & 0.069 & $1.08 \%$ & 0.032 & $0.49 \%$ & 0.017 & $0.2 \%$ & 0.011 & $0.17 \%$ \\
\hline Std Dev & & 0.005 & $0.07 \%$ & 0.005 & $0.08 \%$ & 0.005 & $0.0 \%$ & 0.002 & $0.03 \%$ \\
\hline 95\% Error & & 0.078 & $1.22 \%$ & 0.042 & $0.66 \%$ & 0.027 & $0.4 \%$ & 0.014 & $0.22 \%$ \\
\hline DS2-11 & 40 & 0.445 & $1.4 \%$ & 0.132 & $0.4 \%$ & 0.054 & $0.2 \%$ & 0.035 & $0.1 \%$ \\
\hline DS2-12 & 40 & 0.476 & $1.5 \%$ & 0.108 & $0.3 \%$ & 0.055 & $0.2 \%$ & 0.038 & $0.1 \%$ \\
\hline DS2-13 & 40 & 0.434 & $1.4 \%$ & 0.123 & $0.4 \%$ & 0.069 & $0.2 \%$ & 0.039 & $0.1 \%$ \\
\hline DS2-14 & 40 & 0.567 & $1.8 \%$ & 0.182 & $0.6 \%$ & 0.099 & $0.3 \%$ & 0.058 & $0.2 \%$ \\
\hline DS2-15 & 40 & 0.418 & $1.3 \%$ & 0.106 & $0.3 \%$ & 0.102 & $0.3 \%$ & 0.054 & $0.2 \%$ \\
\hline Average & & 0.468 & $1.46 \%$ & 0.130 & $0.41 \%$ & 0.076 & $0.2 \%$ & 0.045 & $0.14 \%$ \\
\hline Std Dev & & 0.059 & $0.19 \%$ & 0.031 & $0.10 \%$ & 0.023 & $0.0 \%$ & 0.010 & $0.03 \%$ \\
\hline 95\% Error & & 0.587 & $1.83 \%$ & 0.192 & $0.60 \%$ & 0.122 & $0.3 \%$ & 0.066 & $0.21 \%$ \\
\hline
\end{tabular}

Table-2: Time Distortion Errors after Corrections for HEAF Cameras

$\%$ Is percent of actual record length (Actual $=80 \%$ of Sweep Setting).

The $95 \%$ error is average error plus two standard deviations. 
Distortions on space can be measured from records like Fig. 2. However, we get better mapping data without modulating the laser beam. Fig. 3 shows this type of record.

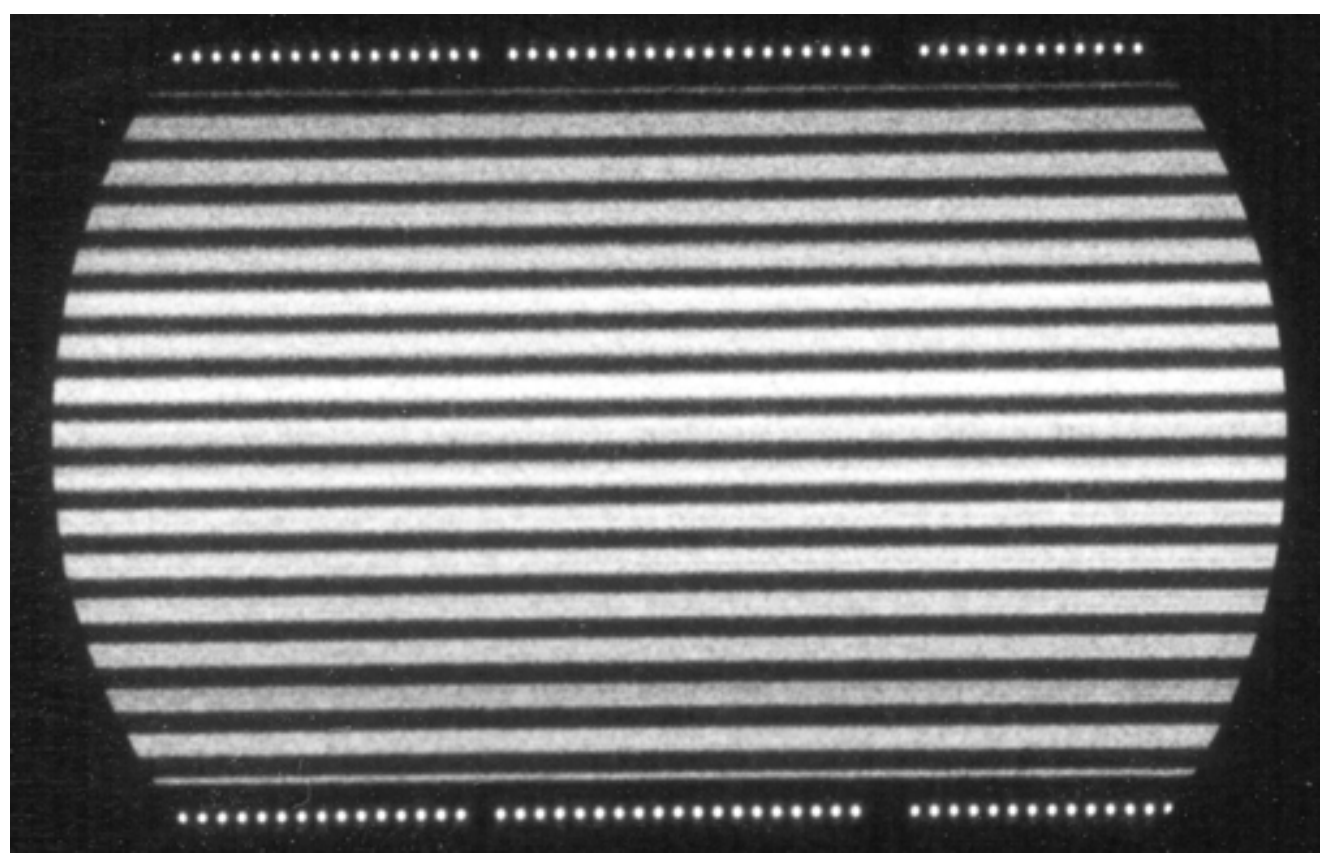

Figure 3. Space Distortion Measurement Record

The effects of space distortion on velocity measurements are considered for two interferometer cavity lengths, called short and long. Long cavities (60 $\mathrm{mm}$ to $65 \mathrm{~mm}$ ) are used to measure velocities that are about $3 \mathrm{~mm} /$ microsecond or less. Higher velocities are usually measured using a short cavity (30-mm). The long and short cavity interferometer setups use different lens sets (the spherical and relay lens sets in Fig. 1). To test the effectiveness of space distortion corrections we use a crystal placed within a $1.984 \mathrm{GHz}$ microwave cavity. This phase modulates the transmitted 532-nM wavelength laser beam to get a record as shown in Fig. 4. 


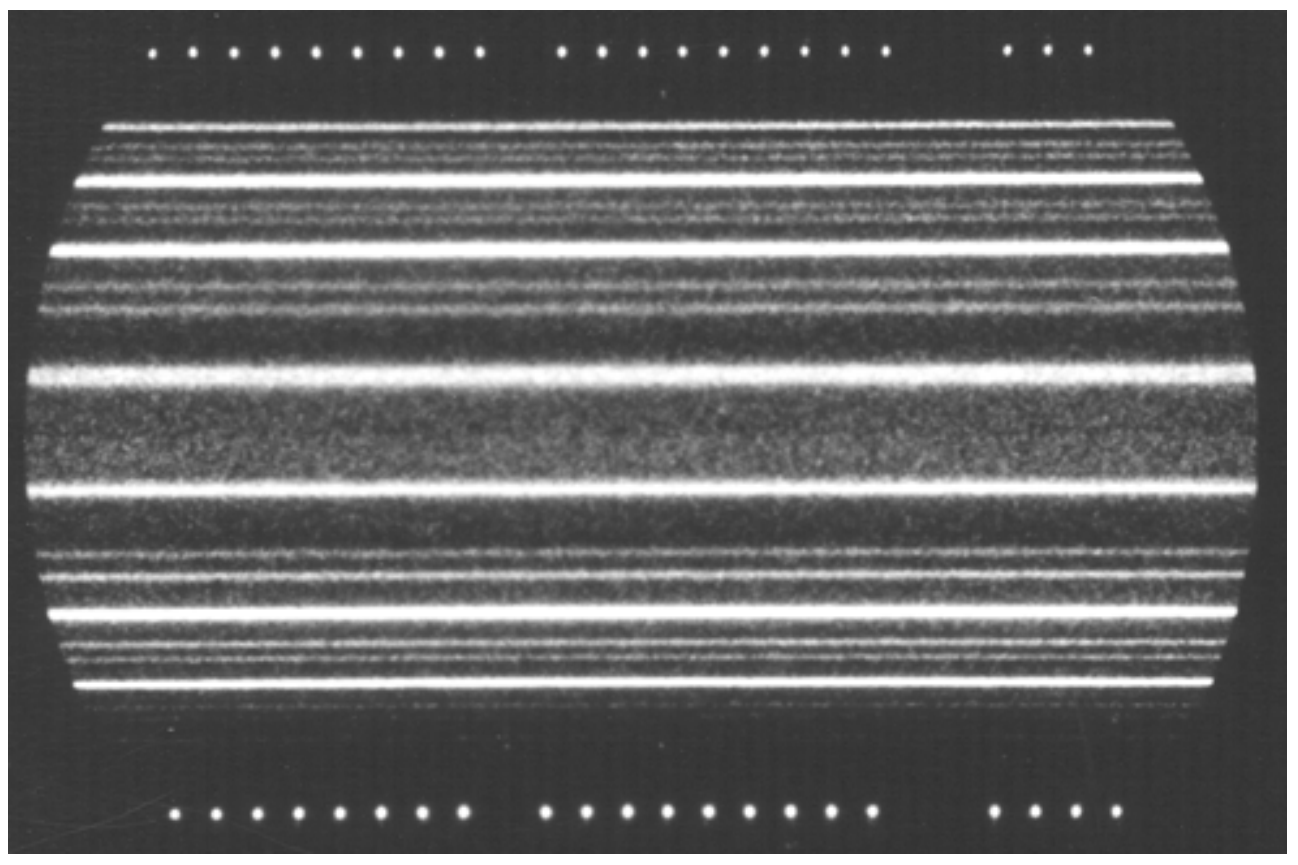

Figure 4. Velocity Error Measurement Record

The bright fringes are not modulated and they represent UN-Doppler shifted fringes. The weaker modulated fringes represent Doppler shifts of plus and minus 0.528 $\mathrm{mm} /$ microsecond and modulation errors are sufficiently small to neglect for our purposes. Records like Fig. 4 are analyzed to get the velocity error data listed in Table-3 through Table-8. The term "Double Records" refers to the method of recording light from two different surface locations onto a single streak camera (using the top and bottom halves of a film record for two fringe sets). 


$\begin{array}{lccccccc}\text { CAMERA } & \begin{array}{c}\text { CAMERA } \\ \text { SER. NO. }\end{array} & \begin{array}{c}\text { TOP/BOT } \\ \text { SWEP } \\ \text { Microsec. }\end{array} & \begin{array}{c}\text { FIDS } \\ \text { mm/Microsec. }\end{array} & \begin{array}{c}\text { THREE } \\ \text { FIDS } \\ \text { mm/Microsec. }\end{array} & \begin{array}{l}\text { FULL } \\ \text { MAP } \\ \text { mm/Microsec. }\end{array} \\ \text { DS1-2 } & 8 & 0.013 & 1.0 \% & 0.020 & 1.5 \% & 0.014 & 1.1 \% \\ \text { DS1-4 } & 8 & 0.009 & 0.7 \% & 0.008 & 0.6 \% & 0.009 & 0.7 \% \\ \text { DS1-5 } & 8 & 0.012 & 0.9 \% & 0.010 & 0.8 \% & 0.010 & 0.8 \% \\ \text { DS2-1 } & 8 & 0.014 & 1.1 \% & 0.014 & 1.1 \% & 0.011 & 0.8 \% \\ \text { DS2-3 } & 8 & 0.007 & 0.5 \% & 0.007 & 0.5 \% & 0.006 & 0.5 \% \\ \text { DS2-5 } & 8 & 0.014 & 1.1 \% & 0.011 & 0.8 \% & 0.009 & 0.7 \% \\ \text { DS2-6 } & 8 & 0.009 & 0.7 \% & 0.009 & 0.7 \% & 0.007 & 0.5 \% \\ \text { DS2-7 } & 8 & 0.006 & 0.5 \% & 0.008 & 0.6 \% & 0.006 & 0.5 \% \\ \text { DS2-8 } & 8 & 0.012 & 0.9 \% & 0.014 & 1.1 \% & 0.012 & 0.9 \% \\ \text { DS2-10 } & 8 & 0.010 & 0.8 \% & 0.013 & 1.0 \% & 0.005 & 0.4 \% \\ & & & & & & & \\ \text { Average } & & 0.011 & 0.80 \% & 0.011 & 0.86 \% & 0.009 & 0.67 \% \\ \text { Std Dev } & & 0.003 & 0.21 \% & 0.004 & 0.30 \% & 0.003 & 0.22 \% \\ \text { 95\% Error } & & 0.016 & 1.22 \% & 0.019 & 1.45 \% & 0.015 & 1.11 \% \\ & & & & & & & \\ \text { DS1-2 } & & & & & & & \\ \text { DS1-4 } & 40 & 0.018 & 1.4 \% & 0.019 & 1.4 \% & 0.012 & 0.9 \% \\ \text { DS1-5 } & 40 & 0.009 & 0.7 \% & 0.009 & 0.7 \% & 0.016 & 1.2 \% \\ \text { DS2-1 } & 40 & 0.009 & 0.7 \% & 0.011 & 0.8 \% & 0.004 & 0.3 \% \\ \text { DS2-3 } & 40 & 0.019 & 1.4 \% & 0.022 & 1.7 \% & 0.010 & 0.8 \% \\ \text { DS2-5 } & 40 & 0.008 & 0.6 \% & 0.009 & 0.7 \% & 0.011 & 0.8 \% \\ \text { DS2-6 } & 40 & 0.014 & 1.1 \% & 0.011 & 0.8 \% & 0.009 & 0.7 \% \\ \text { DS2-7 } & 40 & 0.010 & 0.8 \% & 0.011 & 0.8 \% & 0.019 & 1.4 \% \\ \text { DS2-8 } & 40 & 0.008 & 0.6 \% & 0.009 & 0.7 \% & 0.008 & 0.6 \% \\ \text { DS2-10 } & 40 & 0.015 & 1.1 \% & 0.009 & 0.7 \% & 0.018 & 1.4 \% \\ & 40 & 0.011 & 0.8 \% & 0.016 & 1.2 \% & 0.009 & 0.7 \% \\ \text { Average } & & & & & & \\ \text { Std Dev } & & 0.012 & 0.91 \% & 0.013 & 0.95 \% & 0.012 & 0.87 \% \\ \text { 95\% Error } & & 0.004 & 0.31 \% & 0.005 & 0.35 \% & 0.005 & 0.36 \% \\ & & 0.020 & 1.53 \% & 0.022 & 1.66 \% & 0.021 & 1.59 \%\end{array}$

Table-3: Velocity Errors after Corrections for Site 300 Cameras Short Cavity Interferometer, Double Records are not used. $\%$ is percent of fringe constant $(1.33 \mathrm{~mm} /$ microsecond $)$.

The $95 \%$ error is average error plus two standard deviations. 


\begin{tabular}{|c|c|c|c|c|c|c|c|}
\hline $\begin{array}{l}\text { CAMERA } \\
\text { SER. NO. }\end{array}$ & $\begin{array}{l}\text { CAMERA } \\
\text { SWEEP } \\
\text { Microsec. }\end{array}$ & \multicolumn{2}{|c|}{$\begin{array}{l}\text { TOP/BOT } \\
\text { FIDS } \\
\text { mm/Microsec c. }\end{array}$} & \multicolumn{2}{|c|}{$\begin{array}{l}\text { THREE } \\
\text { FIDS } \\
\text { mm/Microsec. }\end{array}$} & \multicolumn{2}{|c|}{$\begin{array}{l}\text { FULL } \\
\text { MAP } \\
\text { mm/Microsec. }\end{array}$} \\
\hline DS1-2 & 8 & 0.071 & $5.3 \%$ & 0.019 & $1.4 \%$ & 0.025 & $1.9 \%$ \\
\hline DS1-4 & 8 & 0.044 & $3.3 \%$ & 0.020 & $1.5 \%$ & 0.015 & $1.1 \%$ \\
\hline DS1-5 & 8 & 0.054 & $4.1 \%$ & 0.021 & $1.6 \%$ & 0.022 & $1.7 \%$ \\
\hline DS2-1 & 8 & 0.019 & $1.4 \%$ & 0.019 & $1.4 \%$ & 0.021 & $1.6 \%$ \\
\hline DS2-3 & 8 & 0.016 & $1.2 \%$ & 0.015 & $1.1 \%$ & 0.011 & $0.8 \%$ \\
\hline DS2-5 & 8 & 0.039 & $2.9 \%$ & 0.021 & $1.6 \%$ & 0.021 & $1.6 \%$ \\
\hline DS2-6 & 8 & 0.023 & $1.7 \%$ & 0.019 & $1.4 \%$ & 0.015 & $1.1 \%$ \\
\hline DS2-7 & 8 & 0.011 & $0.8 \%$ & 0.011 & $0.8 \%$ & 0.013 & $1.0 \%$ \\
\hline DS2-8 & 8 & 0.025 & $1.9 \%$ & 0.018 & $1.4 \%$ & 0.026 & $2.0 \%$ \\
\hline DS2-10 & 8 & 0.025 & $1.9 \%$ & 0.012 & $0.9 \%$ & 0.008 & $0.6 \%$ \\
\hline Average & & 0.033 & $2.46 \%$ & 0.018 & $1.32 \%$ & 0.018 & $1.33 \%$ \\
\hline Std Dev & & 0.019 & $1.43 \%$ & 0.004 & $0.27 \%$ & 0.006 & $0.46 \%$ \\
\hline 95\% Error & & 0.071 & $5.31 \%$ & 0.025 & $1.86 \%$ & 0.030 & $2.25 \%$ \\
\hline DS1-2 & 40 & 0.053 & $4.0 \%$ & 0.017 & $1.3 \%$ & 0.015 & $1.1 \%$ \\
\hline DS1-4 & 40 & 0.055 & $4.1 \%$ & 0.014 & $1.1 \%$ & 0.021 & $1.6 \%$ \\
\hline DS1-5 & 40 & 0.043 & $3.2 \%$ & 0.018 & $1.4 \%$ & 0.014 & $1.1 \%$ \\
\hline DS2-1 & 40 & 0.042 & $3.2 \%$ & 0.023 & $1.7 \%$ & 0.013 & $1.0 \%$ \\
\hline DS2-3 & 40 & 0.020 & $1.5 \%$ & 0.013 & $1.0 \%$ & 0.017 & $1.3 \%$ \\
\hline DS2-5 & 40 & 0.058 & $4.4 \%$ & 0.022 & $1.7 \%$ & 0.016 & $1.2 \%$ \\
\hline DS2-6 & 40 & 0.032 & $2.4 \%$ & 0.014 & $1.1 \%$ & 0.022 & $1.7 \%$ \\
\hline DS2-7 & 40 & 0.011 & $0.8 \%$ & 0.011 & $0.8 \%$ & 0.017 & $1.3 \%$ \\
\hline DS2-8 & 40 & 0.024 & $1.8 \%$ & 0.024 & $1.8 \%$ & 0.037 & $2.8 \%$ \\
\hline DS2-10 & 40 & 0.029 & $2.2 \%$ & 0.017 & $1.3 \%$ & 0.015 & $1.1 \%$ \\
\hline Average & & 0.037 & $2.76 \%$ & 0.017 & $1.30 \%$ & 0.019 & $1.41 \%$ \\
\hline Std Dev & & 0.016 & $1.20 \%$ & 0.004 & $0.34 \%$ & 0.007 & $0.53 \%$ \\
\hline 95\% Error & & 0.069 & $5.17 \%$ & 0.026 & $1.97 \%$ & 0.033 & $2.46 \%$ \\
\hline
\end{tabular}

Table-4: Velocity Errors after Corrections for Site 300 Cameras

Short Cavity Interferometer, Double Records are used.

$\%$ is percent of fringe constant $(1.33 \mathrm{~mm} /$ microsecond).

The $95 \%$ error is average error plus two standard deviations. 


\begin{tabular}{|c|c|c|c|c|c|c|c|}
\hline $\begin{array}{l}\text { CAMERA } \\
\text { SER. NO. }\end{array}$ & $\begin{array}{l}\text { CAMERA } \\
\text { SWEEP } \\
\text { Microsec. }\end{array}$ & \multicolumn{2}{|c|}{$\begin{array}{l}\text { TOP/BOT } \\
\text { FIDS } \\
\text { mm/Microsec c. }\end{array}$} & \multicolumn{2}{|c|}{$\begin{array}{l}\text { THREE } \\
\text { FIDS } \\
\text { mm/Microsec. }\end{array}$} & \multicolumn{2}{|c|}{$\begin{array}{l}\text { FULL } \\
\text { MAP } \\
\text { mm/Microsec. }\end{array}$} \\
\hline DS1-2 & 8 & 0.011 & $1.7 \%$ & 0.011 & $1.7 \%$ & 0.006 & $0.9 \%$ \\
\hline DS1-4 & 8 & 0.010 & $1.5 \%$ & 0.010 & $1.5 \%$ & 0.009 & $1.4 \%$ \\
\hline DS1-5 & 8 & 0.009 & $1.4 \%$ & 0.010 & $1.5 \%$ & 0.010 & $1.5 \%$ \\
\hline DS2-1 & 8 & 0.013 & $2.0 \%$ & 0.009 & $1.4 \%$ & 0.009 & $1.4 \%$ \\
\hline DS2-3 & 8 & 0.004 & $0.6 \%$ & 0.005 & $0.8 \%$ & 0.004 & $0.6 \%$ \\
\hline DS2-5 & 8 & 0.008 & $1.2 \%$ & 0.006 & $0.9 \%$ & 0.004 & $0.6 \%$ \\
\hline DS2-6 & 8 & 0.009 & $1.4 \%$ & 0.01 & $1.5 \%$ & 0.006 & $0.9 \%$ \\
\hline DS2-7 & 8 & 0.008 & $1.2 \%$ & 0.006 & $0.9 \%$ & 0.010 & $1.5 \%$ \\
\hline DS2-8 & 8 & 0.006 & $0.9 \%$ & 0.006 & $0.9 \%$ & 0.005 & $0.8 \%$ \\
\hline DS2-10 & 8 & 0.006 & $0.9 \%$ & 0.005 & $0.8 \%$ & 0.005 & $0.8 \%$ \\
\hline Average & & 0.008 & $1.26 \%$ & 0.008 & $1.17 \%$ & 0.007 & $1.02 \%$ \\
\hline Std Dev & & 0.003 & $0.4 \%$ & 0.002 & $0.4 \%$ & 0.002 & $0.4 \%$ \\
\hline 95\% Error & & 0.014 & $2.1 \%$ & 0.013 & $1.9 \%$ & 0.012 & $1.8 \%$ \\
\hline DS1-2 & 40 & 0.017 & $2.6 \%$ & 0.013 & $2.0 \%$ & 0.006 & $0.9 \%$ \\
\hline DS1-4 & 40 & 0.009 & $1.4 \%$ & 0.007 & $1.1 \%$ & 0.004 & $0.6 \%$ \\
\hline DS1-5 & 40 & 0.010 & $1.5 \%$ & 0.012 & $1.8 \%$ & 0.010 & $1.5 \%$ \\
\hline DS2-1 & 40 & 0.017 & $2.6 \%$ & 0.017 & $2.6 \%$ & 0.014 & $2.1 \%$ \\
\hline DS2-3 & 40 & 0.012 & $1.8 \%$ & 0.008 & $1.2 \%$ & 0.008 & $1.2 \%$ \\
\hline DS2-5 & 40 & 0.007 & $1.1 \%$ & 0.007 & $1.1 \%$ & 0.007 & $1.1 \%$ \\
\hline DS2-6 & 40 & 0.009 & $1.4 \%$ & 0.007 & $1.1 \%$ & 0.007 & $1.1 \%$ \\
\hline DS2-7 & 40 & 0.008 & $1.2 \%$ & 0.006 & $0.9 \%$ & 0.005 & $0.8 \%$ \\
\hline DS2-8 & 40 & 0.007 & $1.1 \%$ & 0.009 & $1.4 \%$ & 0.005 & $0.8 \%$ \\
\hline DS2-10 & 40 & 0.006 & $0.9 \%$ & 0.006 & $0.9 \%$ & 0.004 & $0.6 \%$ \\
\hline Average & & 0.010 & $1.53 \%$ & 0.009 & $1.38 \%$ & 0.007 & $1.05 \%$ \\
\hline Std Dev & & 0.004 & $0.6 \%$ & 0.004 & $0.5 \%$ & 0.003 & $0.5 \%$ \\
\hline 95\% Error & & 0.018 & $2.7 \%$ & 0.016 & $2.5 \%$ & 0.013 & $2.0 \%$ \\
\hline
\end{tabular}

Table-5: Velocity Errors after Corrections for Site 300 Cameras Long Cavity Interferometer, Double Records are not used. $\%$ is percent of fringe constant $(0.665 \mathrm{~mm} / \mathrm{microsecond})$.

The $95 \%$ error is average error plus two standard deviations. 


\begin{tabular}{|c|c|c|c|c|c|c|c|}
\hline $\begin{array}{l}\text { CAMERA } \\
\text { SER. NO. }\end{array}$ & $\begin{array}{l}\text { CAMERA } \\
\text { SWEEP } \\
\text { Microsec. }\end{array}$ & \multicolumn{2}{|c|}{$\begin{array}{l}\text { TOP/BOT } \\
\text { FIDS } \\
\text { mm/Microsec. }\end{array}$} & \multicolumn{2}{|c|}{$\begin{array}{l}\text { THREE } \\
\text { FIDS } \\
\text { mm/Microsec. }\end{array}$} & \multicolumn{2}{|c|}{$\begin{array}{l}\text { FULL } \\
\text { MAP } \\
\text { mm/Microsec. }\end{array}$} \\
\hline DS1-2 & 8 & 0.035 & $5.3 \%$ & 0.019 & $2.9 \%$ & 0.011 & $1.7 \%$ \\
\hline DS1-4 & 8 & 0.030 & $4.5 \%$ & 0.015 & $2.3 \%$ & 0.018 & $2.7 \%$ \\
\hline DS1-5 & 8 & 0.031 & $4.7 \%$ & 0.020 & $3.0 \%$ & 0.021 & $3.2 \%$ \\
\hline DS2-1 & 8 & 0.024 & $3.6 \%$ & 0.016 & $2.4 \%$ & 0.017 & $2.6 \%$ \\
\hline DS2-3 & 8 & 0.010 & $1.5 \%$ & 0.009 & $1.4 \%$ & 0.008 & $1.2 \%$ \\
\hline DS2-5 & 8 & 0.025 & $3.8 \%$ & 0.010 & $1.5 \%$ & 0.009 & $1.4 \%$ \\
\hline DS2-6 & 8 & 0.018 & $2.7 \%$ & 0.018 & $2.7 \%$ & 0.015 & $2.3 \%$ \\
\hline DS2-7 & 8 & 0.011 & $1.7 \%$ & 0.010 & $1.5 \%$ & 0.013 & $2.0 \%$ \\
\hline DS2-8 & 8 & 0.012 & $1.8 \%$ & 0.009 & $1.4 \%$ & 0.009 & $1.4 \%$ \\
\hline DS2-10 & 8 & 0.013 & $2.0 \%$ & 0.008 & $1.2 \%$ & 0.009 & $1.4 \%$ \\
\hline Average & & 0.021 & $3.14 \%$ & 0.013 & $2.02 \%$ & 0.013 & $1.95 \%$ \\
\hline Std Dev & & 0.009 & $1.4 \%$ & 0.005 & $0.7 \%$ & 0.005 & $0.7 \%$ \\
\hline 95\% Error & & 0.039 & $5.9 \%$ & 0.023 & $3.4 \%$ & 0.022 & $3.3 \%$ \\
\hline DS1-2 & 40 & 0.041 & $6.2 \%$ & 0.018 & $2.7 \%$ & 0.015 & $2.3 \%$ \\
\hline DS1-4 & 40 & 0.028 & $4.2 \%$ & 0.010 & $1.5 \%$ & 0.006 & $0.9 \%$ \\
\hline DS1-5 & 40 & 0.033 & $5.0 \%$ & 0.018 & $2.7 \%$ & 0.021 & $3.2 \%$ \\
\hline DS2-1 & 40 & 0.021 & $3.2 \%$ & 0.024 & $3.6 \%$ & 0.022 & $3.3 \%$ \\
\hline DS2-3 & 40 & 0.023 & $3.5 \%$ & 0.012 & $1.8 \%$ & 0.011 & $1.7 \%$ \\
\hline DS2-5 & 40 & 0.034 & $5.1 \%$ & 0.016 & $2.4 \%$ & 0.015 & $2.3 \%$ \\
\hline DS2-6 & 40 & 0.022 & $3.3 \%$ & 0.012 & $1.8 \%$ & 0.019 & $2.9 \%$ \\
\hline DS2-7 & 40 & 0.012 & $1.8 \%$ & 0.009 & $1.4 \%$ & 0.007 & $1.1 \%$ \\
\hline DS2-8 & 40 & 0.019 & $2.9 \%$ & 0.014 & $2.1 \%$ & 0.011 & $1.7 \%$ \\
\hline DS2-10 & 40 & 0.019 & $2.9 \%$ & 0.012 & $1.8 \%$ & 0.012 & $1.8 \%$ \\
\hline Average & & 0.025 & $3.79 \%$ & 0.015 & $2.18 \%$ & 0.014 & $2.09 \%$ \\
\hline Std Dev & & 0.009 & $1.3 \%$ & 0.005 & $0.7 \%$ & 0.006 & $0.8 \%$ \\
\hline 95\% Error & & 0.043 & $6.4 \%$ & 0.024 & $3.5 \%$ & 0.025 & $3.8 \%$ \\
\hline
\end{tabular}

Table-6: Velocity Errors after Corrections for Site 300 Cameras Long Cavity Interferometer, Double Records are used. $\%$ is percent of fringe constant $(0.665 \mathrm{~mm} / \mathrm{microsecond})$.

The $95 \%$ error is average error plus two standard deviations. 


\begin{tabular}{|c|c|c|c|c|c|c|c|}
\hline $\begin{array}{l}\text { CAMERA } \\
\text { SER. NO. }\end{array}$ & $\begin{array}{l}\text { CAMERA } \\
\text { SWEEP } \\
\text { Microsec. }\end{array}$ & \multicolumn{2}{|c|}{$\begin{array}{l}\text { TOP/BOT } \\
\text { FIDS } \\
\text { mm/Microsec. }\end{array}$} & \multicolumn{2}{|c|}{$\begin{array}{l}\text { THREE } \\
\text { FIDS } \\
\text { mm/Microsec. }\end{array}$} & \multicolumn{2}{|c|}{$\begin{array}{l}\text { FULL } \\
\text { MAP } \\
\text { mm/Microsec. }\end{array}$} \\
\hline DS2-11 & 8 & 0.006 & $0.9 \%$ & 0.006 & $0.9 \%$ & 0.010 & $1.5 \%$ \\
\hline DS2-12 & 8 & 0.013 & $1.9 \%$ & 0.013 & $1.9 \%$ & 0.016 & $2.3 \%$ \\
\hline DS2-13 & 8 & 0.008 & $1.2 \%$ & 0.008 & $1.2 \%$ & 0.009 & $1.3 \%$ \\
\hline DS2-14 & 8 & 0.011 & $1.6 \%$ & 0.011 & $1.6 \%$ & 0.013 & $1.9 \%$ \\
\hline DS2-15 & 8 & 0.010 & $1.5 \%$ & 0.010 & $1.5 \%$ & 0.008 & $1.2 \%$ \\
\hline Average & & 0.010 & $1.40 \%$ & 0.010 & $1.40 \%$ & 0.011 & $1.63 \%$ \\
\hline Std Dev & & 0.003 & $0.4 \%$ & 0.003 & $0.4 \%$ & 0.003 & $0.5 \%$ \\
\hline 95\% Error & & 0.015 & $2.2 \%$ & 0.015 & $2.2 \%$ & 0.018 & $2.6 \%$ \\
\hline DS2-11 & 40 & 0.008 & $1.2 \%$ & 0.008 & $1.2 \%$ & 0.009 & $1.3 \%$ \\
\hline DS2-12 & 40 & 0.007 & $1.0 \%$ & 0.007 & $1.0 \%$ & 0.008 & $1.2 \%$ \\
\hline DS2-13 & 40 & 0.006 & $0.9 \%$ & 0.006 & $0.9 \%$ & 0.008 & $1.2 \%$ \\
\hline DS2-14 & 40 & 0.018 & $2.6 \%$ & 0.018 & $2.6 \%$ & 0.014 & $2.0 \%$ \\
\hline DS2-15 & 40 & 0.011 & $1.6 \%$ & 0.011 & $1.6 \%$ & 0.010 & $1.5 \%$ \\
\hline Average & & 0.010 & $1.46 \%$ & 0.010 & $1.46 \%$ & 0.010 & $1.43 \%$ \\
\hline Std Dev & & 0.005 & $0.7 \%$ & 0.005 & $0.7 \%$ & 0.002 & $0.4 \%$ \\
\hline $95 \%$ Error & & 0.020 & $2.9 \%$ & 0.020 & $2.9 \%$ & 0.015 & $2.2 \%$ \\
\hline
\end{tabular}

\section{Table-7: Velocity Errors after Corrections for HEAF Cameras} Long Cavity Interferometer, Double Records are not used. $\%$ is percent of fringe constant $(0.6868 \mathrm{~mm} /$ microsecond $)$. The $95 \%$ error is average error plus two standard deviations. 


\begin{tabular}{|c|c|c|c|c|c|c|c|}
\hline $\begin{array}{l}\text { CAMERA } \\
\text { SER. NO. }\end{array}$ & $\begin{array}{l}\text { CAMERA } \\
\text { SWEEP } \\
\text { Microsec. }\end{array}$ & \multicolumn{2}{|c|}{$\begin{array}{l}\text { TOP/BOT } \\
\text { FIDS } \\
\text { mm/Microsec. }\end{array}$} & \multicolumn{2}{|c|}{$\begin{array}{l}\text { THREE } \\
\text { FIDS } \\
\text { mm/Microsec. }\end{array}$} & \multicolumn{2}{|c|}{$\begin{array}{l}\text { FULL } \\
\text { MAP } \\
\text { mm/Microsec. }\end{array}$} \\
\hline DS2-11 & 8 & 0.014 & $2.0 \%$ & 0.011 & $1.6 \%$ & 0.016 & $2.3 \%$ \\
\hline DS2-12 & 8 & 0.018 & $2.6 \%$ & 0.019 & $2.8 \%$ & 0.021 & $3.1 \%$ \\
\hline DS2-13 & 8 & 0.015 & $2.2 \%$ & 0.014 & $2.0 \%$ & 0.013 & $1.9 \%$ \\
\hline DS2-14 & 8 & 0.038 & $5.5 \%$ & 0.018 & $2.6 \%$ & 0.014 & $2.0 \%$ \\
\hline DS2-15 & 8 & 0.019 & $2.8 \%$ & 0.024 & $3.5 \%$ & 0.021 & $3.1 \%$ \\
\hline Average & & 0.021 & $3.03 \%$ & 0.017 & $2.50 \%$ & 0.017 & $2.48 \%$ \\
\hline Std Dev & & 0.010 & $1.4 \%$ & 0.005 & $0.7 \%$ & 0.004 & $0.6 \%$ \\
\hline $95 \%$ Error & & 0.040 & $5.9 \%$ & 0.027 & $4.0 \%$ & 0.025 & $3.6 \%$ \\
\hline DS2-11 & 40 & 0.018 & $2.6 \%$ & 0.011 & $1.6 \%$ & 0.017 & $2.5 \%$ \\
\hline DS2-12 & 40 & 0.019 & $2.8 \%$ & 0.019 & $2.8 \%$ & 0.013 & $1.9 \%$ \\
\hline DS2-13 & 40 & 0.010 & $1.5 \%$ & 0.006 & $0.9 \%$ & 0.012 & $1.7 \%$ \\
\hline DS2-14 & 40 & 0.051 & $7.4 \%$ & 0.021 & $3.1 \%$ & 0.017 & $2.5 \%$ \\
\hline DS2-15 & 40 & 0.019 & $2.8 \%$ & 0.021 & $3.1 \%$ & 0.019 & $2.8 \%$ \\
\hline Average & & 0.023 & $3.41 \%$ & 0.016 & $2.27 \%$ & 0.016 & $2.27 \%$ \\
\hline Std Dev & & 0.016 & $2.3 \%$ & 0.007 & $1.0 \%$ & 0.003 & $0.4 \%$ \\
\hline 95\% Error & & 0.055 & $8.0 \%$ & 0.029 & $4.2 \%$ & 0.022 & $3.1 \%$ \\
\hline
\end{tabular}

Table-8: Velocity Errors after Corrections for HEAF Cameras

Long Cavity Interferometer, Double Records are used.

$\%$ is percent of fringe constant $(0.6868 \mathrm{~mm} /$ microsecond $)$.

The $95 \%$ error is average error plus two standard deviations.

\section{Distortion over Calendar Time Study}

Three streak cameras were mapped by placing a precision ruling at the slit plane so that comparisons could be made to 1992 records of the same type. Table-9 makes comparisons by applying years 1992 and
2000 maps to correct 1992 distortion records. We see that the year 2000 time correction map is no better than just using fresh top and bottom fiducials.

$\begin{array}{ccc}\text { Mod-Sn } & \text { Tube \# } & \text { Rec. Year } \\ \text { Ds2-1 } & \text { LS-131 } & 1992 \\ \text { Ds2-5 } & \text { LS-138 } & 1992 \\ \text { Ds2-3 } & \text { LS-129 } & 1992\end{array}$

\begin{tabular}{ccccc} 
& & \multicolumn{3}{c}{ CORRECTION TYPE } \\
& None & Top/Bot & 1992 Map & 2000 Map \\
& 0.044 & 0.025 & 0.013 & 0.029 \\
& 0.054 & 0.033 & 0.011 & 0.038 \\
& 0.049 & 0.030 & 0.011 & 0.026 \\
\cline { 2 - 5 } Average & 0.049 & 0.029 & 0.012 & 0.031 \\
\hline Std Dev & 0.005 & 0.004 & 0.001 & 0.006
\end{tabular}

Table-9: Time Correction of Year 1992 Records Using 1992 and 2000 Correction Records Sweep Rate $=10$ microseconds. Units are microseconds.

The ruling is at the slit. 
Similar comparisons were made for records that are more recent. Table-10 applies years 1999 and 2000 correction maps to year 2000 records. The older map is worse. For correcting 1999 and 2000 records, the ruling was placed back at the shaper optics. Space distortion studies showed similar signs of gradual distortion change over calendar time (see Table-11 and Table-12).

$\begin{array}{ccc}\text { Mod-Sn } & \text { Tube \# } & \text { Rec. Year } \\ \text { Ds2-1 } & \text { LS-131 } & 2000 \\ \text { Ds2-5 } & \text { LS-138 } & 2000 \\ \text { Ds2-3 } & \text { LS-129 } & 2000\end{array}$

\begin{tabular}{ccccc} 
& & \multicolumn{3}{c}{ CORRECTION TYPE } \\
& None & Top/Bot & 1999 Map & 2000 Map \\
& 0.056 & 0.032 & 0.022 & 0.016 \\
& 0.076 & 0.021 & 0.014 & 0.011 \\
& 0.042 & 0.024 & 0.018 & 0.012 \\
\cline { 2 - 5 } Average & 0.058 & 0.026 & 0.018 & 0.013 \\
\cline { 2 - 5 } Std Dev & 0.017 & 0.006 & 0.004 & 0.003
\end{tabular}

Table-10: Time Correction of Year 2000 Records Using 1999 and 2000 Correction Records Sweep Rate $=10$ microseconds. Units are microseconds. The ruling is at the shaper optics.

\begin{tabular}{lccccccc} 
Mod-Sn & Tube \# & Rec. Year & & None & Top/Bot & 1992 Map & 2000 Map \\
Ds2-1 & LS-131 & 1992 & & 0.007 & 0.007 & 0.003 & 0.009 \\
Ds2-5 & LS-138 & 1992 & & 0.009 & 0.009 & 0.003 & 0.009 \\
Ds2-3 & LS-129 & 1992 & & 0.007 & 0.007 & 0.002 & 0.008 \\
\cline { 4 - 8 } & & & Average & 0.008 & 0.008 & 0.003 & 0.009 \\
\cline { 3 - 7 } & & & Std Dev & 0.001 & 0.001 & 0.001 & 0.001
\end{tabular}

Table-11: Space Correction of Year 1992 Records Using 1992 and 2000 Correction Records Sweep Rate $=10$ microseconds. Units are fraction of slit length (record height). The ruling is at the slit.

$\begin{array}{ccc}\text { Mod-Sn } & \text { Tube \# } & \text { Rec. Year } \\ \text { Ds2-1 } & \text { LS-131 } & 1999 \\ \text { Ds2-5 } & \text { LS-138 } & 1999 \\ \text { Ds2-3 } & \text { LS-129 } & 1999\end{array}$

\begin{tabular}{ccccc} 
& None & Top/Bot & 1999 Map & 2000 Map \\
& 0.011 & 0.011 & 0.007 & 0.013 \\
& 0.011 & 0.011 & 0.008 & 0.011 \\
& 0.012 & 0.009 & 0.008 & 0.009 \\
\hline Average & 0.011 & 0.010 & 0.008 & 0.011 \\
\hline Std Dev & 0.001 & 0.001 & 0.001 & 0.002
\end{tabular}

Table-12: Space Correction of Year 1999 Records Using 1999 and 2000 Correction Records Sweep Rate $=10$ microseconds. Units are fraction of slit length (record height). The ruling is at the shaper optics.

\section{Analysis of the Records}

The records discussed above are digitized along each fringe by using Barry Jacoby's routine that performs a least squares curve fit on the optical density variation across a fringe (or fiducial). Fringe profiles are fit to a cubic while timing fid (fiducial) dots are fit to quadratic. Following digitization, the analysis begins by establishing a horizontal time axis along a linear spatial fit to the bottom row of fid dots. Time is then linearly scaled to the 
actual time interval between the lower first and last fid dots. A reference grid is then overlaid and centered to the lower-middle fid dot. It spans out in equal increments of the actual fid period to match a count of the spaces between fid dots (whether dimmed or bright). Fig. 5 shows an overlaid reference grid where small circles represent the distorted film record times. Timing errors are calculated as deviations from the reference grid. These errors are plotted and curve fit as shown in Fig. 6 . Each curve of Fig. 6 represents a row of fid dots from Fig. 5.

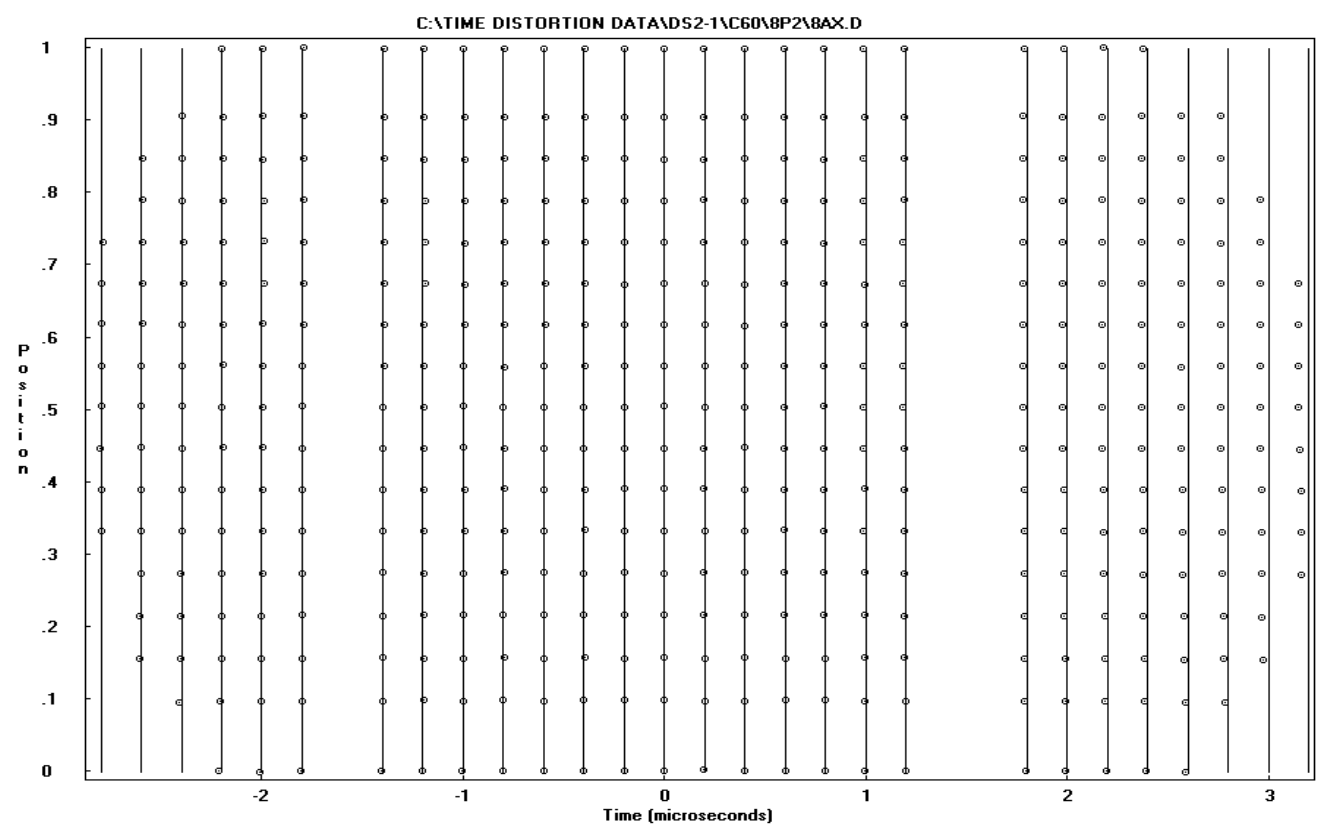

Figure 5. Time Reference Overlay Grid onto Scaled Data

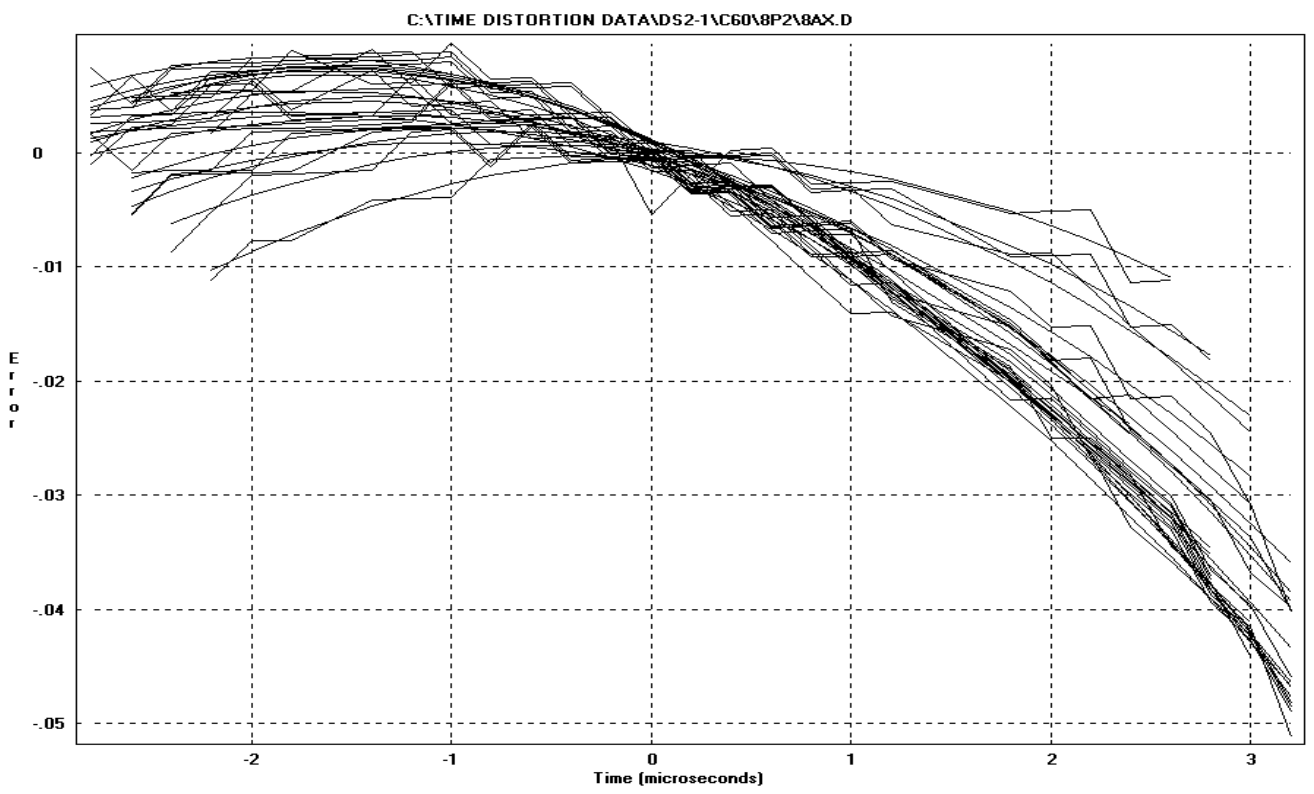

Figure 6. Time Error Plots for Scaled Data Only 
The curves for the top and bottom fids are averaged and this new curve serves as a correction curve for the first stage of distortion corrections on all data fields of the record. A new set of error curves are now plotted and fit as shown in Fig. 7. The top and bottom fid errors are now corrected but the middle regions still have distortion errors.

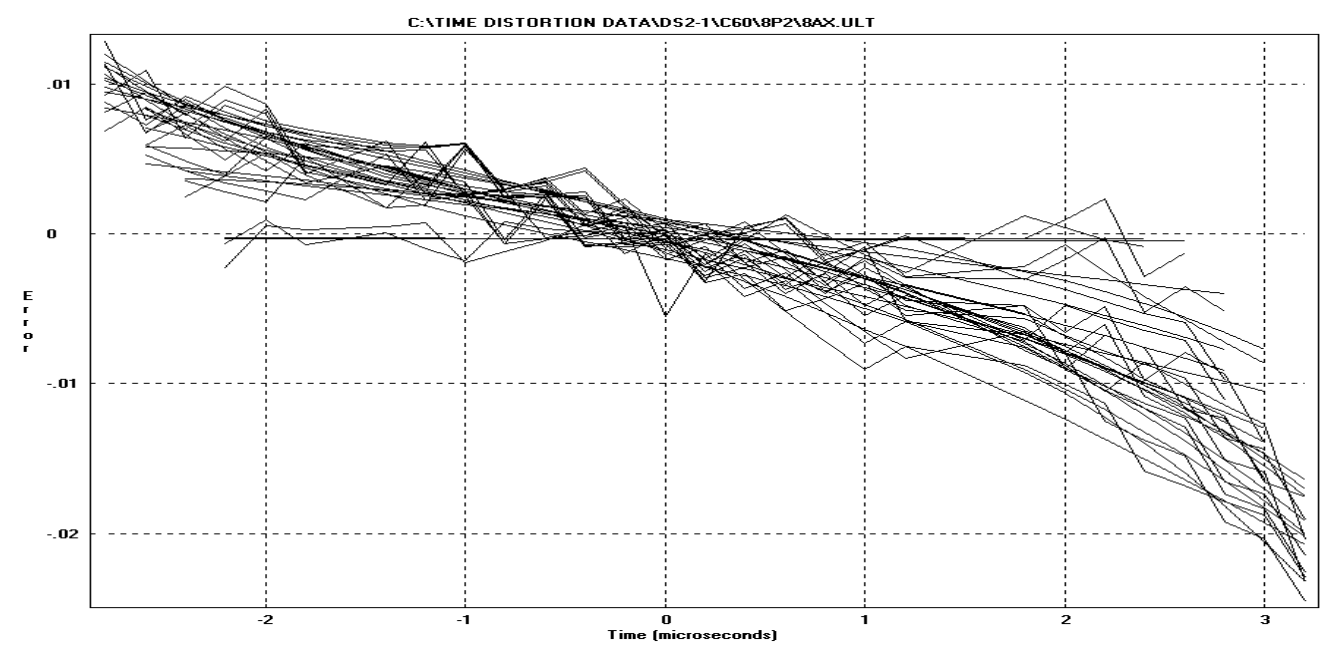

Figure 7. Time Error Plots for Corrections Using Only Top and Bottom Fids

For the full map correction, we now use each error curve from Fig. 7 (except for the top and bottom fid curves) to correct distortions in the inner regions. Each curve now takes on a third coordinate which is the relative distance of a given fid row from the lower end of the slit (vertical axis of Fig. 5).
All regions between fid rows are linearly interpolated from the two nearest fid rows. Fig. 8 plots and fits the final errors after the full map corrections. The time errors of Table- 1 and Table-2 include the random noise errors outside of the smooth curves.

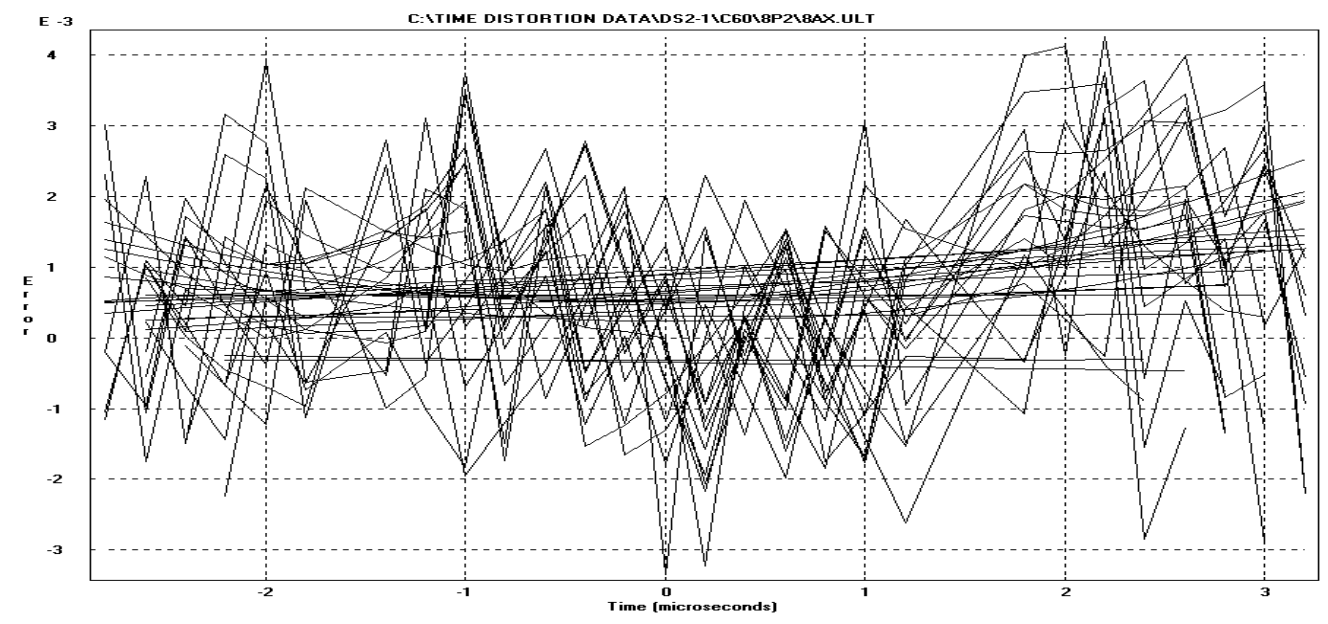

Figure 8. Time Error Plots for Corrections Using a Full Map 
Time corrections for using three fids are evaluated by removing all fid rows from the digitized record except for the top, bottom and middle rows. Since there is zero lens distortion at the center of the record, this provides the same record as having all three fids attached to the streak camera slit. For analysis, the same correction (using only top and bottom fids) described above is performed first. Next, the smooth correction curve for the middle fid row is used to correct all regions except for the top and bottom fid row positions. Interpolation is used so that the corrections have maximum strength for middle regions and diminish to zero as we work our way to the top (or bottom) fid row. Errors for the three-fid correction method are shown in Fig. 9. Although the corrections are not quite as good as with the full map, the corrections are derived from the same record as the experiment. A mid slit timing fid does not block any useable light from the experiment.

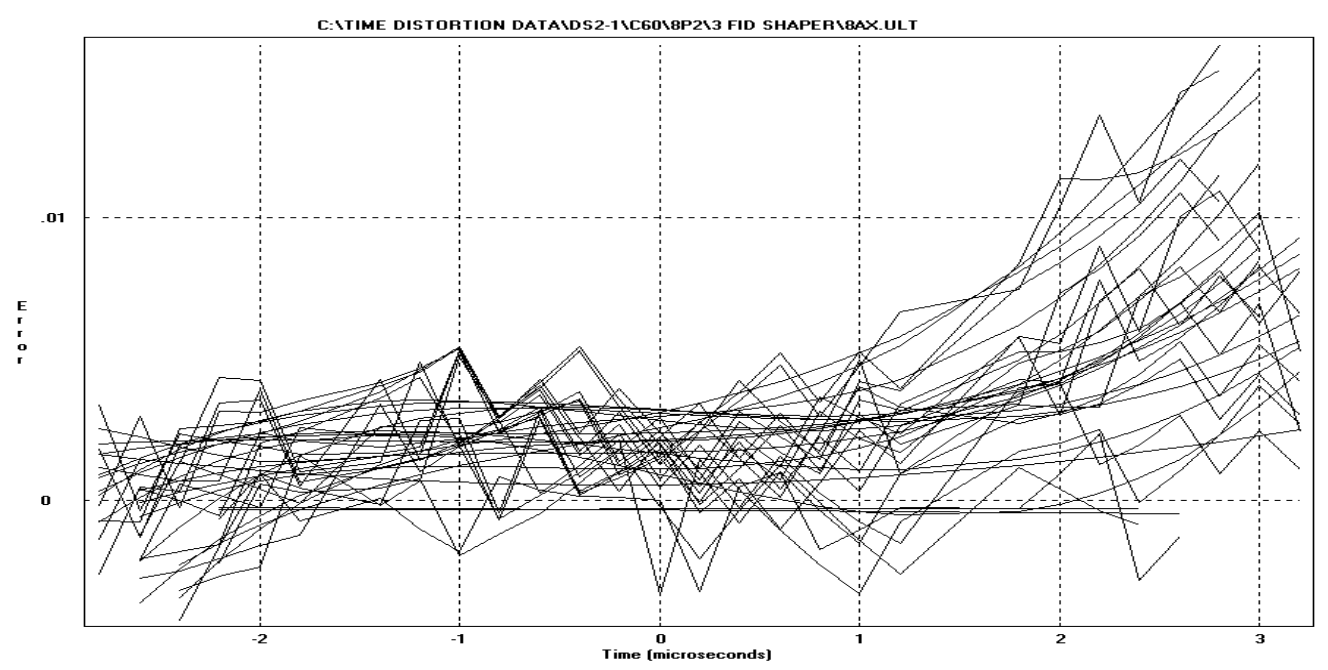

Figure 9. Time Error Plots for Corrections Using Only Three Fids

Space distortion corrections are made next. Fig. 10 and Fig. 11 show the overlay of equal spaced lines that are used to measure differences from the nominal (zero distortion). The lines are overlaid onto a digitized film record like the one in Fig. 3. The average line spacing is determined as the distance between the uppermost and lowermost lines (not the top and bottom fids) divided by the number of spaces between lines. The overlay thus fits the outermost lines best. Errors are deviations from the nominal reference lines in units of fractions of the slit length. Curves fit to these errors are used for space corrections just as they were for the time corrections. 


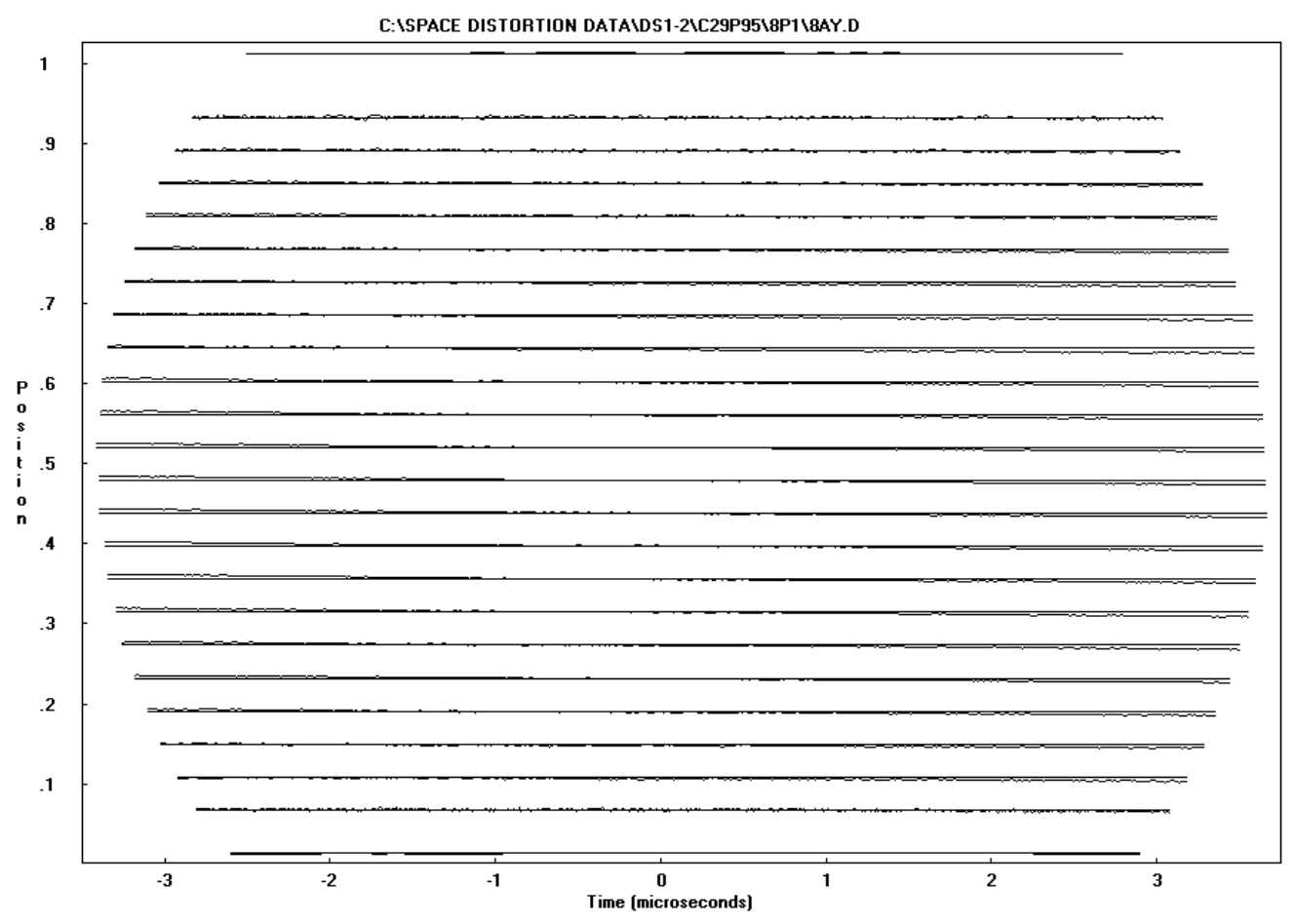

Figure 10. Space Reference Overlay Lines onto Scaled Data

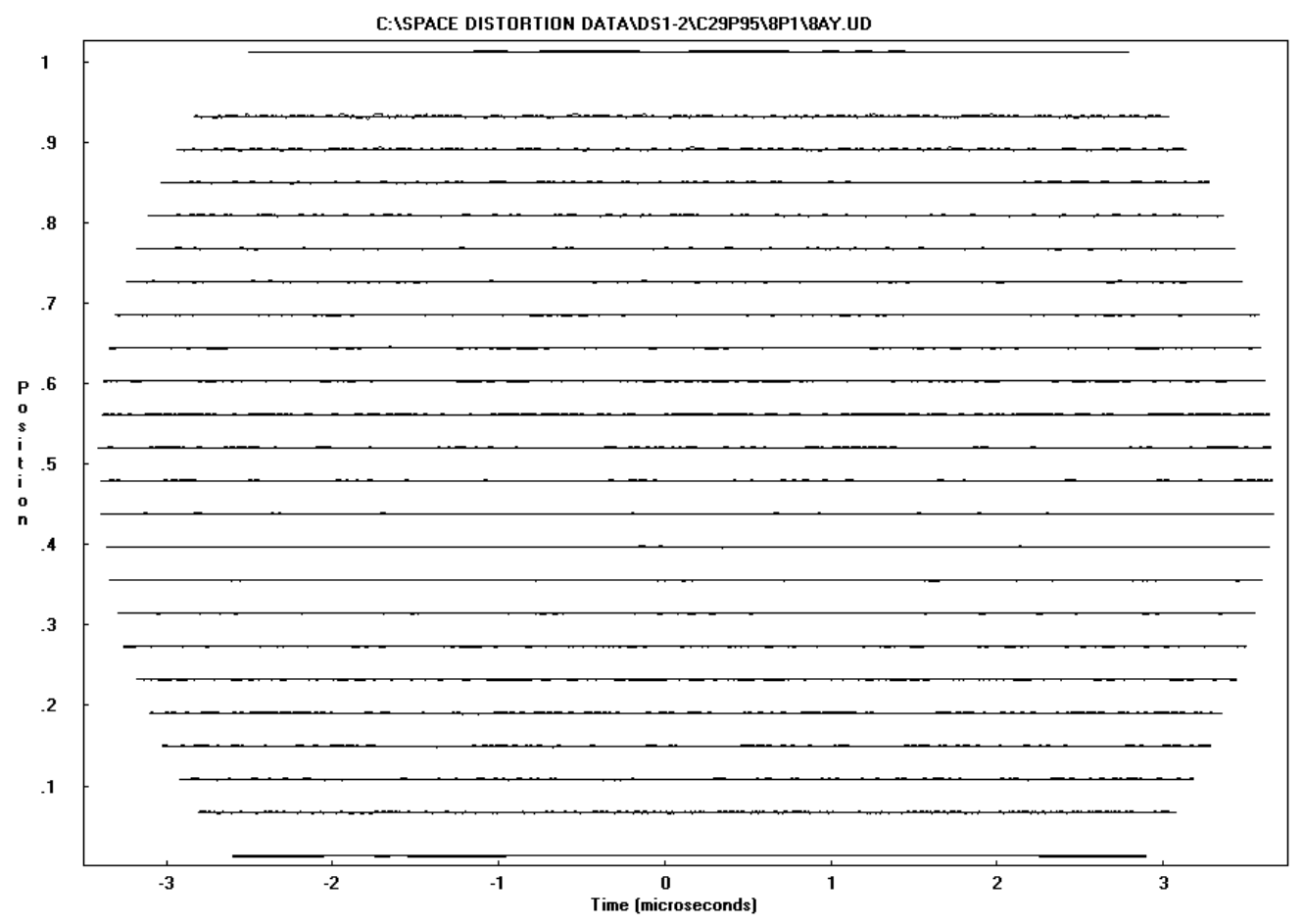

Figure 11. Space Reference Overlay Lines onto Full Map Corrected Data 
We test the effectiveness of space corrections by analyzing records obtained using the phase modulated laser beam discussed earlier (see Fig. 4). For each analysis, a table such as Table-13 is produced to evaluate the effectiveness of distortion corrections. The maximum errors listed in the table are calculated from an average of the maximum errors found from curves fitted to at least two analysis records.

\begin{tabular}{|c|c|c|c|}
\hline $\begin{array}{l}\text { NON DOU } \\
\text { FILE }\end{array}$ & AVG ERROR & MAX ERROR & NOISE STD DEV \\
\hline 8AF1.ERR & -0.0036 & -0.0129 & 0.0065 \\
\hline 8AF1A.ERR & -0.0090 & -0.0143 & 0.0080 \\
\hline 8AF1B.ERR & 0.0023 & 0.0109 & 0.0093 \\
\hline 8AF2.ERR & 0.0045 & 0.0137 & 0.0080 \\
\hline 8AF2A.ERR & -0.0013 & -0.0169 & 0.0080 \\
\hline 8AF2B.ERR & 0.0108 & 0.0214 & 0.0131 \\
\hline \multicolumn{4}{|c|}{ DOUBLE RECORDS CALIBRATION: ONLY TOP RECORDS ARE USED } \\
\hline FILE & AVG ERROR & MAX ERROR & NOISE STD DEV \\
\hline 8AF1.ERR & -0.0017 & -0.0472 & 0.0092 \\
\hline 8AF1A.ERR & -0.0099 & -0.0490 & 0.0110 \\
\hline 8AF1B.ERR & 0.0074 & 0.0467 & 0.0124 \\
\hline 8AF2.ERR & 0.0102 & 0.0565 & 0.0105 \\
\hline 8AF2A.ERR & -0.0041 & -0.0535 & 0.0086 \\
\hline 8AF2B.ERR & 0.0262 & 0.0699 & 0.0171 \\
\hline \multicolumn{4}{|c|}{ DOUBLE RECORDS CALIBRATION: ONLY BOTTOM RECORDS ARE USED } \\
\hline FILE & AVG ERROR & MAX ERROR & NOISE STD DEV \\
\hline 8AF1.ERR & -0.0053 & -0.0670 & 0.0100 \\
\hline 8AF1A.ERR & -0.0082 & -0.0669 & 0.0125 \\
\hline 8AF1B.ERR & -0.0022 & 0.0633 & 0.0146 \\
\hline 8AF2.ERR & -0.0024 & -0.0751 & 0.0120 \\
\hline 8AF2A.ERR & 0.0019 & -0.0804 & 0.0149 \\
\hline 8AF2B.ERR & -0.0066 & -0.0641 & 0.0191 \\
\hline
\end{tabular}

Table-13: Measured Velocity Errors for Camera DS1-2 before Correction

Sweep Rate $=8$ microseconds. Units are $\mathrm{mm} /$ microsecond.

Data are not corrected for distortion. Fringe Const $=1.329082$.

$\mathrm{F} 1$ and $\mathrm{F} 2$ on the left columns represent the two frequency shifts (plus and minus) from the un-shifted (bright) fringes of Fig. 4. The $A$ and $B$ refer to the most inner and next most inner fringes from mid record. If neither $A$ or $B$ is indicated then that row refers to the average of the two most inner fringe sets and this value is used in the summary tables 3-8. All of this was chosen to represent the data fields that we normally read for analysis on an actual shot record. Table-14 results from applying a full map distortion correction on the same record. 


\begin{tabular}{|c|c|c|c|}
\hline FILE & AVG ERROR & MAX ERROR & NOISE STD DEV \\
\hline 8AF1.ERR & 0.0024 & 0.0068 & 0.0067 \\
\hline 8AF1A.ERR & -0.0060 & -0.0079 & 0.0082 \\
\hline 8AF1B.ERR & 0.0116 & 0.0148 & 0.0095 \\
\hline 8AF2.ERR & 0.0097 & 0.0157 & 0.0081 \\
\hline 8AF2A.ERR & 0.0052 & 0.0086 & 0.0082 \\
\hline 8AF2B.ERR & 0.0147 & 0.0238 & 0.0133 \\
\hline DOUBLE REC & RDS CALIBRAT & TOP RECORD & \\
\hline FILE & AVG ERROR & MAX ERROR & NOISE STD DEV \\
\hline 8AF1.ERR & 0.0056 & 0.0109 & 0.0091 \\
\hline 8AF1A.ERR & -0.0063 & -0.0088 & 0.0111 \\
\hline 8AF1B.ERR & 0.0187 & 0.0249 & 0.0130 \\
\hline 8AF2.ERR & 0.0232 & 0.0268 & 0.0102 \\
\hline 8AF2A.ERR & 0.0076 & 0.0132 & 0.0079 \\
\hline 8AF2B.ERR & 0.0404 & 0.0460 & 0.0166 \\
\hline DOUBLE RE & RDS CALIBRAT & BOTTOM REC & USED \\
\hline FILE & AVG ERROR & MAX ERROR & NOISE STD DEV \\
\hline 8AF1.ERR & -0.0007 & -0.0029 & 0.0097 \\
\hline 8AF1A.ERR & -0.0058 & -0.0074 & 0.0123 \\
\hline 8AF1B.ERR & 0.0050 & 0.0101 & 0.0142 \\
\hline 8AF2.ERR & -0.0050 & -0.0256 & 0.0116 \\
\hline 8AF2A.ERR & 0.0031 & -0.0237 & 0.0140 \\
\hline 8AF2B.ERR & -0.0133 & -0.0298 & 0.0194 \\
\hline
\end{tabular}

Table-14: Measured Velocity Errors for Camera DS1-2 after Correction Sweep Rate $=8$ microseconds. Units are $\mathrm{mm} /$ microsecond. Data are corrected for distortion. Fringe Const $=1.329082$.

The noise standard deviation is tabulated to indicate the noise amplitude (same units) in comparison to to the measured errors.

Fig. 12 and Fig. 13 show how the actual data plots look for the bottom double record errors tabulated in Table-13 and Table-14 respectively. The horizontal line is the calibration velocity $(0.528 \mathrm{~mm} / \mathrm{microsecond})$. It is calculated from the equation $V=\Delta f(\lambda / 2)$ where $\Delta f$ is the crystal modulation frequency $(1.984 \mathrm{GHz})$ and $\lambda$ is the laser wavelength $(532 \mathrm{nM})$. Velocity errors tabulated in the tables are calculated as the difference of smooth curves (from the calibration velocity) and do not include noise contributions. Notice that the tilted (distorted and noisy) lines in Fig. 10 are the cause of some large velocity errors in Fig. 12 (when double records are used). When double records are not used, these errors tend to cancel out of the calculations. That is why the uncorrected velocities are as accurate as the corrected velocities, when double records are not used. 


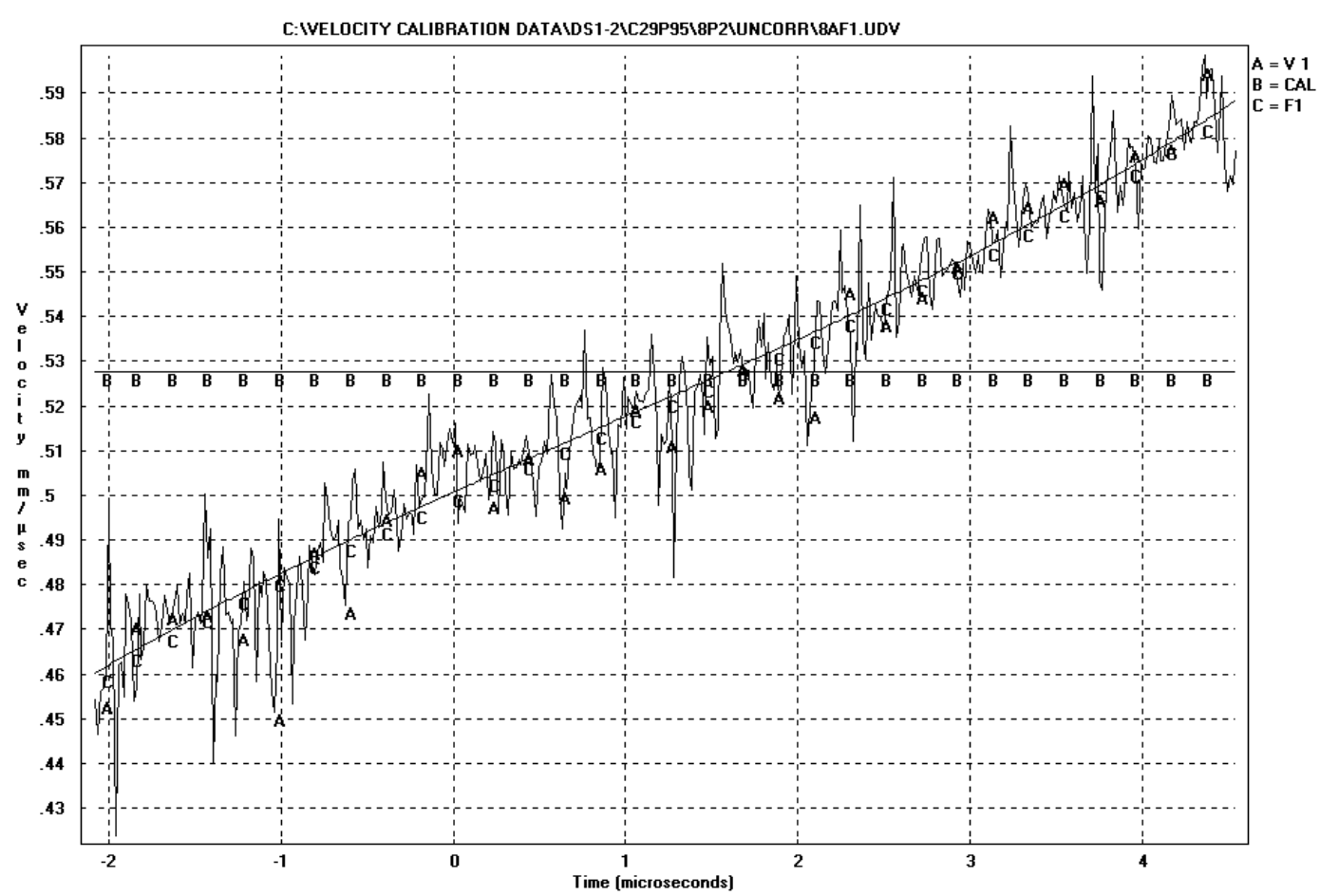

Figure 12. Uncorrected Velocity Overlaid onto Calibration Velocity

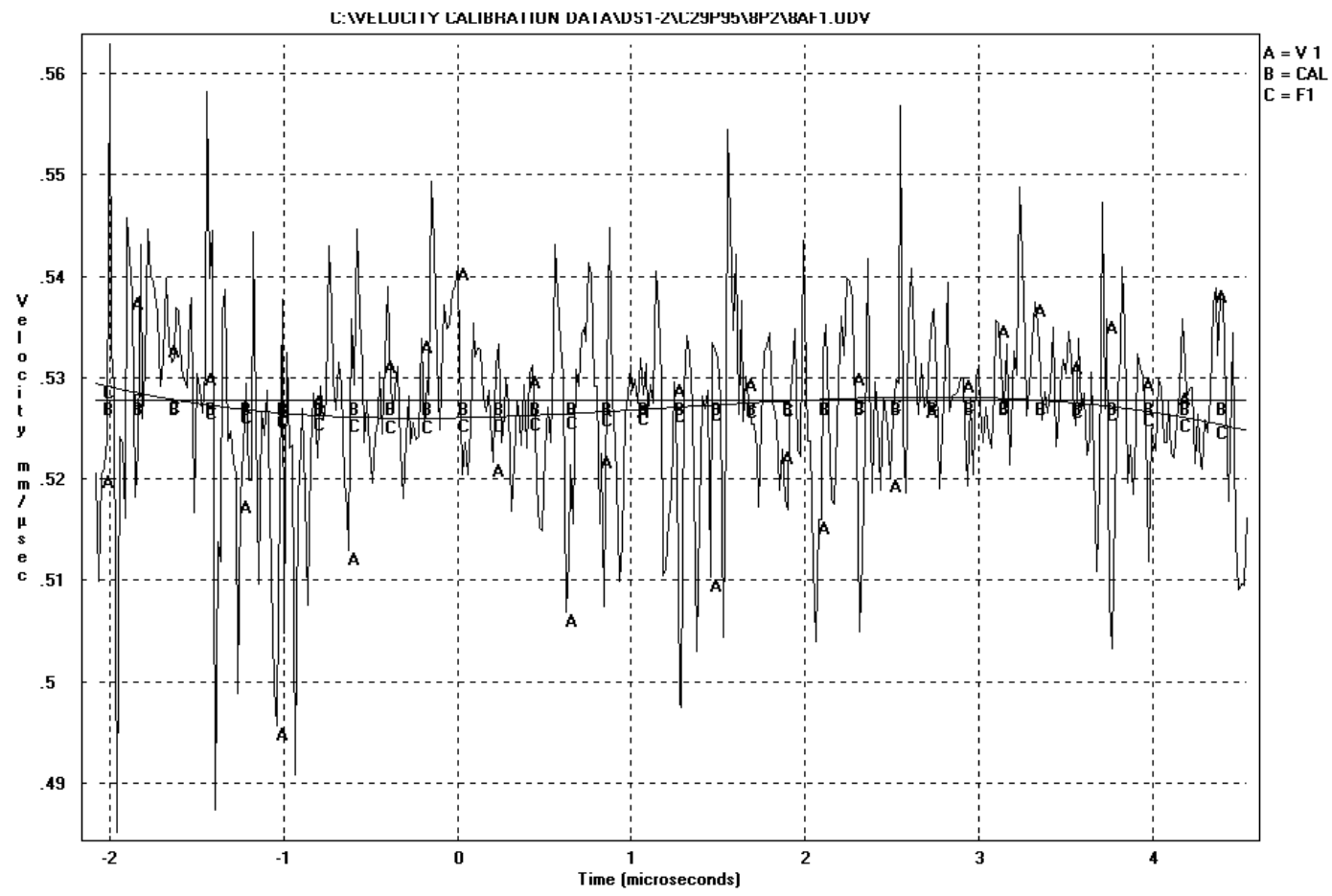

Figure 13. Corrected Velocity Overlaid onto Calibration Velocity 


\section{Conclusions and Recommendations}

We have shown that our velocimetry data can be corrected for distortions in timing and velocity by using full distortion maps that are a significant improvement over the current two fiducial (fid) methods. However, the map corrections will require time consuming recalibrations at least annually. Currently, we spend about two weeks to calibrate five cameras. During busy shot schedules, these calibrations will not likely be done because of the extra man day costs and the time delays in producing the final corrected data.

While the three-fid corrections are less effective than the full map corrections, they are still a very useful improvement over the two-fid corrections currently in use. The three-fid timing errors are only about half as large as the two-fid timing errors (based on fast record writing for minimizing timing errors).
When we do not use our double record capabilities, the three-fid velocity errors are about the same as that for both the two-fid errors and the full map errors. When using the double record capabilities, the three-fid velocity errors are about half that for the two-fid velocity errors. They are about equal to velocity errors of the full map corrections. Keep in mind that the three-fid corrections are also fresh for every record as they are recorded along with the shot data.

We therefore recommend that one streak camera be modified for a three-fid slit plate test vehicle. We can then prove that there are no surprises about our conclusions before installing three-fid slit plates into our remaining cameras. Any shots that justify the extra precision can be scheduled for the extra time and costs of a full map calibration which will be completed for the required cameras just prior to (or just after) the shot date.

\section{References}

1. C. F. McMillan, D. R. Goosman, N. L. Parker, L. L. Steinmetz, H. H. Chau, T. Huen, R. K. Whipkey, and S. J. Perry, Rev. Sci. Instrum. 59, 1 (1988).

2. David Goosman, George Avara, Lloyd Steinmetz, Ching Lai, Stephen Perry, "Manybeam velocimeter for fast surfaces", $22^{\text {nd }}$ International Congress on High-Speed Photography and Photonics, SPIE 2869, 1070 (1996). 